\title{
Supporting Information: Techno-Economic Analysis and Life Cycle Assessment of Five VGO Processing \\ Pathways in China
}

Xin Zhou ${ }^{1}$, Qiang Zhai ${ }^{2 *}$, Chunlan Chen ${ }^{1}$, Hao Yan $^{1}$, Xiaobo Chen ${ }^{1}$, Hui

$$
\text { Zhao }^{1 *} \text {, Chaohe Yang }{ }^{*}
$$

(1. State Key Laboratory of Heavy Oil Processing, China University of Petroleum, Qingdao, Shandong 266580, China; 2. School of Mechanical, Electrical \& Information Engineering, Shandong University, Weihai, Shandong 264209, China)

*Corresponding author: E-mail: zhaohui@upc.edu.cn; zhaiqiang@sdu.edu.cn; yangch@upc.edu.cn. 
Table S1. Properties of Daqing VGO as the feedstock of all the process designs.

\begin{tabular}{cc|cc}
\hline Item & value & Item & value \\
\hline $\boldsymbol{\rho}\left(\mathbf{2 0}{ }^{\circ} \mathrm{C}\right) /\left(\mathbf{k g} \cdot \boldsymbol{m}^{-\mathbf{3}}\right)$ & 854.0 & Paraffin & 5.07 \\
$\boldsymbol{\omega}($ Carbon residue $) / \%$ & 0.25 & Naphthenes & 81.57 \\
$\boldsymbol{\omega}($ Element content $) / \%$ & & Aromatics & 13.36 \\
$\mathrm{C}$ & 86.78 & Distillation curve & \\
$\mathrm{H}$ & 13.11 & $\mathrm{IBP} / 10 \%$ & $358^{\circ} \mathrm{C} / 367^{\circ} \mathrm{C}$ \\
$\mathrm{N}$ & 0.03 & $30 \% / 50 \%$ & $395^{\circ} \mathrm{C} / 420^{\circ} \mathrm{C}$ \\
$\mathrm{S}$ & 0.08 & $70 \% / 90 \%$ & $451^{\circ} \mathrm{C} / 483^{\circ} \mathrm{C}$ \\
$\boldsymbol{\omega}($ group compositions $) / \%$ & & $95 \% / \mathrm{FBP} \%$ & $492^{\circ} \mathrm{C} / 500^{\circ} \mathrm{C}$ \\
\hline
\end{tabular}


Table S2. The structural dimension parameters (geometry) of FCC unit (2.0 Mt/y).

\begin{tabular}{|c|c|}
\hline Item & the FCC unit \\
\hline \multicolumn{2}{|l|}{ Riser } \\
\hline Total length/m & 54.4 \\
\hline Diameter $/ \mathrm{m}$ & 1.15 \\
\hline \multicolumn{2}{|l|}{ Riser Termination Zone } \\
\hline Length/m & 3.28 \\
\hline Diameter/m & 6.54 \\
\hline \multicolumn{2}{|l|}{ Catalyst Stripper } \\
\hline Height/m & 9.27 \\
\hline Diameter/m & 3.11 \\
\hline Annulus Diameter/m & 1.25 \\
\hline \multicolumn{2}{|l|}{ Regenerator } \\
\hline Dense Bed Height $/ \mathrm{m}$ & 5.84 \\
\hline Dense Bed Diameter/m & 8.57 \\
\hline Dilute Phase Diameter $/ \mathrm{m}$ & 11.45 \\
\hline Interface Diameter $/ \mathrm{m}$ & 11.45 \\
\hline Cyclone Inlet Height $/ \mathrm{m}$ & 19.63 \\
\hline Cyclone Inlet Diameter $/ \mathrm{m}$ & 3.04 \\
\hline Cyclone Outlet Diameter/m & 1.59 \\
\hline
\end{tabular}


Table S3. The structural dimension parameters (geometry) of DCC unit (2.0 Mt/y).

\begin{tabular}{|c|c|}
\hline Item & the DCC unit \\
\hline \multicolumn{2}{|l|}{ Riser } \\
\hline Total length/m & 56.7 \\
\hline Diameter/m & 0.99 \\
\hline \multicolumn{2}{|l|}{ Riser Termination Zone } \\
\hline Length/m & 3.27 \\
\hline Diameter/m & 6.47 \\
\hline \multicolumn{2}{|l|}{ Catalyst Stripper } \\
\hline Height $/ \mathrm{m}$ & 9.33 \\
\hline Diameter/m & 3.04 \\
\hline Annulus Diameter/m & 1.05 \\
\hline \multicolumn{2}{|l|}{ Regenerator } \\
\hline Dense Bed Height $/ \mathrm{m}$ & 5.87 \\
\hline Dense Bed Diameter/m & 8.50 \\
\hline Dilute Phase Diameter $/ \mathrm{m}$ & 11.25 \\
\hline Interface Diameter $/ \mathrm{m}$ & 11.25 \\
\hline Cyclone Inlet Height/m & 19.23 \\
\hline Cyclone Inlet Diameter $/ \mathrm{m}$ & 2.98 \\
\hline Cyclone Outlet Diameter/m & 1.52 \\
\hline
\end{tabular}


Table S4. The structural dimension parameters (geometry) of TMP unit (2.0 Mt/y).

\begin{tabular}{|c|c|}
\hline Item & the TMP unit \\
\hline \multicolumn{2}{|l|}{ Riser1 } \\
\hline Total length/m & 28.5 \\
\hline Diameter $/ \mathrm{m}$ & 0.79 \\
\hline \multicolumn{2}{|l|}{ Riser2 } \\
\hline Total length/m & 29.4 \\
\hline Diameter $/ \mathrm{m}$ & 0.65 \\
\hline \multicolumn{2}{|l|}{ Riser Termination Zone } \\
\hline Length $/ \mathrm{m}$ & 2.40 \\
\hline Diameter $/ \mathrm{m}$ & 6.50 \\
\hline \multicolumn{2}{|l|}{ Catalyst Stripper } \\
\hline Height $/ \mathrm{m}$ & 7.58 \\
\hline Diameter $/ \mathrm{m}$ & 3.30 \\
\hline Annulus Diameter/m & 3.30 \\
\hline \multicolumn{2}{|l|}{ Regenerator } \\
\hline Dense Bed Height $/ \mathrm{m}$ & 5.09 \\
\hline Dense Bed Diameter/m & 8.20 \\
\hline Dilute Phase Diameter/m & 9.30 \\
\hline Interface Diameter $/ \mathrm{m}$ & 9.30 \\
\hline Cyclone Inlet Height $/ \mathrm{m}$ & 14.55 \\
\hline Cyclone Inlet Diameter $/ \mathrm{m}$ & 1.78 \\
\hline Cyclone Outlet Diameter/m & 1.78 \\
\hline
\end{tabular}


Table S5. Process parameters of the FCC, DCC and HTMP processing pathways.

\begin{tabular}{cccc}
\hline Items & FCC & DCC & HTMP \\
\hline Riser outlet temperature $/{ }^{\circ} \mathrm{C}$ & 490 & 556 & $510^{\mathrm{a} / 536^{\mathrm{b}}}$ \\
$\begin{array}{c}\text { Steam to Total Feed Ratio } \\
\text { Dense Bed Temperature } /{ }^{\circ} \mathrm{C}\end{array}$ & 0.05 & 0.07 & 0.06 \\
Equilibrium MAT $/ \%$ & 680 & 703 & 690 \\
Reactor Pressure $/ \mathrm{kPa}$ & 67 & 68 & 66 \\
Regenerator Pressure $/ \mathrm{kPa}$ & 210 & 140 & 120 \\
\hline
\end{tabular}
a: The first riser outlet temperature.
b: The second riser outlet temperature. 
Table S6. The calibration parameters of the FCC, DCC and HTMP processing pathways.

\begin{tabular}{cccc}
\hline Item & FCC & DCC & HTMP \\
\hline Activity on Pathways to C Lump & 0.79 & 2.14 & 2.84 \\
Activity on Pathways to G Lump & 0.27 & 0.78 & 1.75 \\
Activity on Pathways to L Lump & 2.06 & 4.30 & 2.11 \\
Metals Coke Activity & $1.4 \mathrm{e}-4$ & $2.8 \mathrm{e}-4$ & $2.2 \mathrm{e}-4$ \\
Catalyst Deactivation Factor & 0.79 & 0.79 & 0.79 \\
Catalyst Surface Area Parameter & -10.72 & -9.54 & -10.72 \\
Effluent per Mass of Catalyst into Stripper & 104.82 & 215.9 & 137.75 \\
Stripper Parameter & 1.63 & 0.92 & 1.91 \\
H to C Ratio for Coke & 0.90 & 0.97 & 0.89 \\
Coke Burn Activity & 15.30 & 15.21 & 15.30 \\
CO Heterogeneous Burn Activity & -1.00 & -1.12 & -1.00 \\
CO Homogeneous Burn Activity & 10.99 & 10.58 & 10.99 \\
Heat of Cracking Parameter & -0.028 & -0.011 & -0.060 \\
Kinetic Coke Activity Factor & 0.031 & 0.14 & 0.052 \\
\hline
\end{tabular}


Table S7. The calibration parameters of the LCO hydrotreating unit in the HTMP processing pathway.

\begin{tabular}{cc} 
processing pathway. & \\
\hline Items & hydrotreating reactor \\
\hline Global Activity & 9.25 \\
Saturation Activity & 7.59 \\
HDS Activity & 0.021 \\
HDN Activity & 0.11 \\
Cracking Activity & 0.123 \\
Ring-Opening Activity & 0.006
\end{tabular}


Table S8. Process parameters of the HCR-I unit.

\begin{tabular}{cc}
\hline Items & HCR unit \\
\hline Inlet Temperature of HT Reactor $/{ }^{\circ} \mathrm{C}$ & 360.5 \\
Outlet Temperature of HT Reactor $/{ }^{\circ} \mathrm{C}$ & 389.8 \\
Inlet Temperature of HCR Reactor $/{ }^{\circ} \mathrm{C}$ & 387.6 \\
Outlet Temperature of HCR Reactor $/{ }^{\circ} \mathrm{C}$ & 398.7 \\
Reactor Pressure/MPa & 11.87 \\
Hydrogen/oil ratio & 758.5 \\
\hline
\end{tabular}


Table S9. Process parameters of the CCR unit in the HCR-I processing pathway.

\begin{tabular}{cc}
\hline Items & HCR unit \\
\hline $\operatorname{Rx} 1$ Inlet Temperature $/{ }^{\circ} \mathrm{C}$ & 516.8 \\
$\operatorname{Rx} 2$ Inlet Temperature $/{ }^{\circ} \mathrm{C}$ & 520.5 \\
$\operatorname{Rx} 3$ Inlet Temperature $/{ }^{\circ} \mathrm{C}$ & 516.5 \\
$\operatorname{Rx} 4$ Inlet Temperature $/{ }^{\circ} \mathrm{C}$ & 521.7 \\
Reactor Pressure $/ \mathrm{MPa}$ & 0.45 \\
\hline
\end{tabular}


Table S10. Process parameters of the steam cracking unit.

\begin{tabular}{cc}
\hline Items & steam cracking \\
\hline Coil Outlet Temperature $/{ }^{\circ} \mathrm{C}$ & 850 \\
Steam dilution ratio & 0.48 \\
\hline
\end{tabular}


Table S11. The kinetic equation set of hydrocracking tail oil reactions. ${ }^{1}$

\begin{tabular}{|c|c|c|c|}
\hline $\begin{array}{c}\text { Sequence } \\
\text { number }\end{array}$ & Reactions & $\mathrm{E} /(\mathrm{MJ} / \mathrm{kmol})$ & $\mathrm{k}_{0} / \mathrm{s}^{-1}$ \\
\hline 1 & $\begin{array}{c}\mathrm{HVGO}=0.13 \mathrm{H}_{2}+0.88 \mathrm{CH}_{4}+1.066 \mathrm{C}_{2} \mathrm{H}_{4}+0.235 \mathrm{C}_{2} \mathrm{H}_{6} \\
+0.543 \mathrm{C}_{3} \mathrm{H}_{6}+0.0353 \mathrm{C}_{3} \mathrm{H}_{8}+0.0582 \mathrm{C}_{4} \mathrm{H}_{10}+0.21 \mathrm{C}_{4} \mathrm{H}_{8}+0.221 \mathrm{C}_{4} \mathrm{H}_{6}+0.242 \mathrm{C}_{4}{ }^{\prime} \mathrm{s}\end{array}$ & 219.78 & $6.565 \times 10^{1}$ \\
\hline 2 & $\mathrm{C}_{2} \mathrm{H}_{6}=\mathrm{C}_{2} \mathrm{H}_{4}+\mathrm{H}_{2}$ & 272.58 & $4.652 \times 10^{1}$ \\
\hline 3 & $\mathrm{C}_{3} \mathrm{H}_{6}=\mathrm{C}_{2} \mathrm{H}_{2}+\mathrm{CH}_{4}$ & 273.08 & $7.284 \times 10^{12}$ \\
\hline 4 & $\mathrm{C}_{2} \mathrm{H}_{2}+\mathrm{C}_{2} \mathrm{H}_{4}=\mathrm{C}_{4} \mathrm{H}_{6}$ & 172.47 & $1.026 \times 10^{13}$ \\
\hline 5 & $2 \mathrm{C}_{2} \mathrm{H}_{6}=\mathrm{C}_{3} \mathrm{H}_{8}+\mathrm{CH}_{4}$ & 272.75 & $3.750 \times 10^{12}$ \\
\hline 6 & $\mathrm{C}_{2} \mathrm{H}_{4}+\mathrm{C}_{2} \mathrm{H}_{6}=\mathrm{C}_{3} \mathrm{H}_{6}+\mathrm{CH}_{4}$ & 252.60 & $7.083 \times 10^{1}$ \\
\hline 7 & $\mathrm{C}_{3} \mathrm{H}_{8}=\mathrm{C}_{3} \mathrm{H}_{6}+\mathrm{H}_{2}$ & 214.39 & $5.888 \times 10^{11}$ \\
\hline 8 & $\mathrm{C}_{3} \mathrm{H}_{8}=\mathrm{C}_{2} \mathrm{H}_{4}+\mathrm{CH}_{4}$ & 211.51 & $4.692 \times 10^{1 \mathrm{C}}$ \\
\hline 9 & $\mathrm{C}_{3} \mathrm{H}_{8}+\mathrm{C}_{2} \mathrm{H}_{4}=\mathrm{C}_{2} \mathrm{H}_{6}+\mathrm{C}_{3} \mathrm{H}_{6}$ & 246.87 & $2.536 \times 10^{1}$ \\
\hline 10 & $2 \mathrm{C}_{3} \mathrm{H}_{6}=3 \mathrm{C}_{2} \mathrm{H}_{4}$ & 268.23 & $7.386 \times 10^{13}$ \\
\hline 11 & $2 \mathrm{C}_{3} \mathrm{H}_{6}=0.3 \mathrm{C}_{\mathrm{n}} \mathrm{H}_{2 \mathrm{n}-6}+0.14 \mathrm{C}_{6+}+3 \mathrm{CH}_{4}$ & 237.84 & $2.424 \times 10^{1}$ \\
\hline 12 & $\mathrm{C}_{3} \mathrm{H}_{6}+\mathrm{C}_{2} \mathrm{H}_{6}=1-\mathrm{C}_{4} \mathrm{H}_{8}+\mathrm{CH}_{4}$ & 250.84 & $1.0 \times 10^{14}$ \\
\hline 13 & $\mathrm{n}-\mathrm{C}_{4} \mathrm{H}_{10}=2 \mathrm{C}_{2} \mathrm{H}_{4}+\mathrm{H}_{2}$ & 295.44 & $7.0 \times 10^{14}$ \\
\hline 14 & $\mathrm{n}-\mathrm{C}_{4} \mathrm{H}_{10}=\mathrm{C}_{2} \mathrm{H}_{4}+\mathrm{C}_{2} \mathrm{H}_{6}$ & 256.28 & $4.099 \times 10^{1}$ \\
\hline 15 & $\mathrm{n}-\mathrm{C}_{4} \mathrm{H}_{10}=1-\mathrm{C}_{4} \mathrm{H}_{8}+\mathrm{H}_{2}$ & 260.66 & $1.637 \times 10^{12}$ \\
\hline 16 & $1-\mathrm{C}_{4} \mathrm{H}_{8}=0.41 \mathrm{C}_{\mathrm{n}} \mathrm{H}_{2 \mathrm{n}-6}+0.14 \mathrm{C}_{6+}$ & 212.05 & $2.075 \times 10^{1}$ \\
\hline 17 & $1-\mathrm{C}_{4} \mathrm{H}_{8}=\mathrm{H}_{2}+\mathrm{C}_{4} \mathrm{H}_{6}$ & 209.00 & $1.0 \times 10^{10}$ \\
\hline 18 & $\mathrm{C}_{2} \mathrm{H}_{4}+\mathrm{C}_{4} \mathrm{H}_{6}=\mathrm{B}+2 \mathrm{H}_{2}$ & 144.46 & $8.385 \times 10^{9}$ \\
\hline 19 & $\mathrm{C}_{3} \mathrm{H} 6+\mathrm{C}_{4} \mathrm{H}_{6}=\mathrm{T}+2 \mathrm{H}_{2}$ & 148.98 & $9.74 \times 10^{8}$ \\
\hline 20 & $\mathrm{C}_{4} \mathrm{H}_{6}+1-\mathrm{C}_{4} \mathrm{H}_{8}=\mathrm{EB}+2 \mathrm{H}_{2}$ & 242.31 & $6.4 \times 10^{14}$ \\
\hline 21 & $\mathrm{C}_{4} \mathrm{H}_{6}+\mathrm{C}_{4} \mathrm{H}_{6}=\mathrm{ST}+2 \mathrm{H}_{2}$ & 124.40 & $1.51 \times 10^{9}$ \\
\hline
\end{tabular}


Table S12. Process parameters of the NHT unit.

\begin{tabular}{cc}
\hline Items & HCR unit \\
\hline Internal Diameter $/ \mathrm{m}$ & 2.5 \\
Catalyst Loading $/ \mathrm{kg}$ & 6135 \\
Catalyst Density $/ \mathrm{kg} / \mathrm{m}^{3}$ & 1000 \\
Void Fraction & 0.5 \\
\hline
\end{tabular}


Table S13. Process parameters of the HCR-II unit.

\begin{tabular}{cc}
\hline Items & HCR unit \\
\hline Inlet Temperature of HT Reactor $/{ }^{\circ} \mathrm{C}$ & 374.8 \\
Outlet Temperature of HT Reactor $/{ }^{\circ} \mathrm{C}$ & 397.8 \\
Inlet Temperature of HCR Reactor $/{ }^{\circ} \mathrm{C}$ & 387.6 \\
Outlet Temperature of $\mathrm{HCR}$ Reactor $/{ }^{\circ} \mathrm{C}$ & 398.7 \\
Reactor Pressure/MPa & 16.10 \\
Hydrogen/oil ratio & 1065 \\
\hline
\end{tabular}


Table S14. Process parameters of the CCR unit in the HCR-II processing pathway.

\begin{tabular}{cc}
\hline Items & HCR unit \\
\hline Rx 1 Inlet Temperature $/{ }^{\circ} \mathrm{C}$ & 518 \\
Rx 2 Inlet Temperature $/{ }^{\circ} \mathrm{C}$ & 518 \\
$\mathrm{Rx}$ 3 Inlet Temperature $/{ }^{\circ} \mathrm{C}$ & 518 \\
$\mathrm{Rx} 4$ Inlet Temperature $/{ }^{\circ} \mathrm{C}$ & 522 \\
Reactor Pressure/MPa & 0.38 \\
\hline
\end{tabular}


Table S15. DELTA-BASE vectors for the HCR-I unit in the HCR-I processing pathway.

\begin{tabular}{ccccc}
\hline Items & Base & Watson K & Sulfur \% & API \\
\hline Products $/$ wt $\%$ & & & & \\
Make up $\mathrm{H}_{2}$ & -0.0206 & 0.0159 & 0.0018 & 0.0011 \\
Dry gas & 0.0295 & -0.0183 & 0.0124 & -0.0036 \\
Light naphtha & 0.1330 & -0.0912 & -0.0139 & 0.00436 \\
Heavy naphtha & 0.1564 & -0.0689 & -0.0086 & -0.0074 \\
Diesel & 0.1611 & -0.0607 & 0.0031 & -0.0068 \\
Bottom & 0.5406 & 0.2231 & 0.0050 & 0.0124 \\
Total & 1.000 & -0.0001 & -0.0002 & $6 \mathrm{E}-05$ \\
Properties & & & & \\
Heavy naphtha N+A/\% & 54.1 & -23.181 & 14.561 & -1.7309 \\
\hline
\end{tabular}


Table S16. DELTA-BASE vectors for the CCR unit in the HCR-I processing pathway.

\begin{tabular}{|c|c|c|c|}
\hline \multicolumn{2}{|c|}{ Items } & Base & $\mathrm{N}+\mathrm{A} \%$ \\
\hline \multicolumn{2}{|c|}{$\mathrm{H}_{2}$} & 0.0683 & -0.0014 \\
\hline \multicolumn{2}{|c|}{ Dry gas } & 0.0028 & 0.0001 \\
\hline \multicolumn{2}{|c|}{ LPG } & 0.0577 & -0.0010 \\
\hline \multicolumn{2}{|c|}{ Reformate } & 0.8757 & 0.0023 \\
\hline \multicolumn{2}{|c|}{ Total } & 1.000 & 0.0000 \\
\hline & Benzene & 0.2652 & 0.0005 \\
\hline Reformate & Toluene & 0.0632 & 0.0035 \\
\hline \multirow[t]{2}{*}{ Including: } & Xylene & 0.3189 & 0.0001 \\
\hline & Others & 0.2284 & -0.0018 \\
\hline
\end{tabular}


Table S17. DELTA-BASE vectors for the steam cracking unit in the HCR-I processing pathway.

\begin{tabular}{|c|c|c|c|}
\hline \multicolumn{2}{|c|}{ Items } & Base & Watson K \\
\hline \multicolumn{2}{|c|}{ Fuel gas } & 0.1715 & -0.00280 \\
\hline \multicolumn{2}{|c|}{ Ethylene } & 0.2952 & 0.000793 \\
\hline \multicolumn{2}{|c|}{ Propene } & 0.1651 & 0.000494 \\
\hline \multicolumn{2}{|c|}{ Butadiene } & 0.0702 & -0.00890 \\
\hline \multicolumn{2}{|c|}{$\mathrm{C} 4 \mathrm{~s}$} & 0.0424 & -0.00870 \\
\hline \multicolumn{2}{|c|}{ Pyrolysis gasoline } & 0.1204 & 0.01909 \\
\hline \multicolumn{2}{|c|}{ Pyrolysis diesel } & 0.0670 & $1.4 \mathrm{e}-05$ \\
\hline \multicolumn{2}{|c|}{ Pyrolysis fuel oil } & 0.0682 & $2.3 \mathrm{e}-05$ \\
\hline \multicolumn{2}{|c|}{ Total } & 1.000 & $1.7 \mathrm{e}-05$ \\
\hline \multirow{4}{*}{$\begin{array}{l}\text { Pyrolysis } \\
\text { gasoline } \\
\text { Including: }\end{array}$} & Benzene & 0.0420 & 0.00592 \\
\hline & Toluene & 0.0135 & 0.00477 \\
\hline & Xylene & 0.0166 & 0.00381 \\
\hline & Others & 0.0486 & 0.00459 \\
\hline
\end{tabular}


Table S18. DELTA-BASE vectors for the HCR-II unit in the HCR-II processing pathway.

\begin{tabular}{ccccc}
\hline Items & Base & Watson K & Sulfur \% & API \\
\hline Products/wt $\%$ & & & & \\
Make up $\mathrm{H}_{2}$ & -0.0301 & 0.01908 & 0.00216 & 0.00132 \\
Dry gas & 0.0434 & -0.02379 & 0.01612 & -0.00468 \\
LPG & 0.0395 & 0.02212 & -0.02492 & 0.00301 \\
Light naphtha & 0.1475 & -0.1368 & -0.02085 & 0.00654 \\
Heavy naphtha & 0.4965 & -0.08268 & -0.01032 & -0.00888 \\
Jet & 0.1344 & -0.0607 & 0.0031 & -0.0068 \\
Diesel & 0.1688 & 0.26278 & 0.0347 & 0.00949 \\
Total & 1.000 & 0.00001 & -0.00001 & 0.00000 \\
Properties & & & & \\
Heavy naphtha N+A/\% & 49.7 & -21.045 & 12.547 & -0.6545 \\
\hline
\end{tabular}


Table S19. DELTA-BASE vectors for the CCR unit in the HCR-II processing pathway.

\begin{tabular}{|c|c|c|c|}
\hline \multicolumn{2}{|c|}{ Items } & Base & $\mathrm{N}+\mathrm{A} \%$ \\
\hline \multicolumn{2}{|c|}{$\mathrm{H}_{2}$} & 0.0852 & -0.00168 \\
\hline \multicolumn{2}{|c|}{ Dry gas } & 0.00328 & 0.00013 \\
\hline \multicolumn{2}{|c|}{ LPG } & 0.0477 & -0.0011 \\
\hline \multicolumn{2}{|c|}{ Reformate } & 0.86382 & 0.00264 \\
\hline \multicolumn{2}{|c|}{ Total } & 1.000 & 0 \\
\hline & Benzene & 0.1849 & 0.0006 \\
\hline Reformate & Toluene & 0.1246 & 0.00255 \\
\hline \multirow[t]{2}{*}{ Including: } & Xylene & 0.3257 & 0.00011 \\
\hline & Others & 0.2286 & -0.00062 \\
\hline
\end{tabular}


Table S20. DELTA-BASE vectors for the aromatics complex unit in the HCR-II processing pathway.

\begin{tabular}{ccc}
\hline Items & Base & $\mathrm{N}+\mathrm{A} \%$ \\
\hline $\mathrm{H}_{2}$ & -0.0154 & -0.0013 \\
Dry gas & 0.0686 & 0.00021 \\
Raffinate oil & 0.1220 & -0.001 \\
Ben & 0.1993 & 0.00094 \\
Heavy aromatics & 0.0130 & -0.00024 \\
PX & 0.6125 & 0.00139 \\
Total & 1.000 & 0.0000 \\
\hline
\end{tabular}




\section{Table S21. Refinery feedstock and product prices for different processing pathways}

(Based on Brent crude oil price 80 \$/bbl). ${ }^{2}$

\begin{tabular}{|c|c|c|c|}
\hline Items & Price $(¥ / t)$ & Items & Price $(¥ / t)$ \\
\hline Daqing VGO & 3977.90 & Toluene & 4973.40 \\
\hline Hydrogen a & 8813.15 & Xylene & 4939.33 \\
\hline Reforming hydrogen ${ }^{b}$ & 6489.64 & PX & 6418.54 \\
\hline Dry gas/Fuel gas & 3004.44 & $\mathrm{C}_{9+}$ & 4694.39 \\
\hline Ethylene $^{c}$ & 6182.20 & Raffinate oil & 4487.68 \\
\hline Propylene & 6488.63 & Gasoline & 4715.09 \\
\hline Propane & 4414.23 & HON Gasoline & 5534.32 \\
\hline Butadiene & 8508.66 & Jet fuel & 3927.97 \\
\hline $\mathrm{C}_{4 \mathrm{~s}}$ & 4360.47 & Diesel & 4221.84 \\
\hline $\mathrm{C}_{5}$ & 4427.07 & Pyrolysis Diesel & 3092.61 \\
\hline Light naphtha & 4487.68 & Fuel oil & 3024.47 \\
\hline Benzene & 4956.40 & Slurry & 2660.99 \\
\hline
\end{tabular}

a: The price of hydrogen is calculated on the basis of coal as raw material.

b: The reforming hydrogen price is based on the composition of hydrogen and fuel gas.

c: Ethylene prices have been deducted from the corresponding separation costs. 
Table S22. Cost parameters and assumptions for process economic evaluation (Based on Brent crude oil price $80 \$ / \mathbf{b b l}) .^{3}$

\begin{tabular}{cccc}
\hline Items & Base value & Items & Base value \\
\hline Make up water & $3.02 ¥ / \mathrm{t}$ & Operating time & $8400 \mathrm{~h}$ \\
Recycling cooling water & $0.34 ¥ / \mathrm{t}$ & Production load & $100 \%$ \\
LP Steam & $170.22 ¥ / \mathrm{t}$ & Project construction period & 2 years \\
MP Steam & $200.26 ¥ / \mathrm{t}$ & Salaries & $100000 ¥ /$ person/year \\
Power & $0.9 ¥ / \mathrm{kW} \cdot \mathrm{h})$ & Net salvage rate & $3 \%$ \\
fuel oil/fuel gas & $3000 ¥ / \mathrm{t}$ & Project maintenance and & $3 \%$ of total direct capital \\
interest rate & $8 \%$ & management costs & cost \\
plant life span & 15 years & discounted annual rate & $15 \%$ \\
tax rate & $25 \%$ & Benchmark IRR & \\
\end{tabular}


Table S23. Summary of scale factors for estimating the capital cost.

\begin{tabular}{|c|c|c|c|c|c|}
\hline Plant subsystems & $\begin{array}{l}\text { Capacities in base } \\
\text { cases/(10kt/year) }\end{array}$ & Basic costs/(104 $¥)$ & $\begin{array}{l}\text { Scale factors } \\
\qquad(\alpha)\end{array}$ & $\begin{array}{l}\text { comprehensive } \\
\text { adjustment factor } \\
\text { (f) }\end{array}$ & source \\
\hline FCC & 150 & 52000 & 0.8 & 1.02 & 3 \\
\hline DCC/TMP & 220 & 218992 & 0.8 & 1.02 & 3 \\
\hline HCR-I & 200 & 88353 & 0.8 & 1.02 & 4 \\
\hline HCR-II & 200 & 110441 & 0.8 & 1.02 & 4 \\
\hline CCR & 60 & 427.5 & 0.8 & 1.02 & 5 \\
\hline Steam Cracking & 70 & 337330 & 0.8 & 1.02 & 6 \\
\hline Aromatics Extraction & 30 & 14900 & 0.6 & 1.02 & 6 \\
\hline Aromatics Complex & 100 & 268000 & 0.8 & 1.02 & 6 \\
\hline Gasoline Hydrotreating & 70 & 24780 & 0.8 & 1.02 & 4 \\
\hline LCO Hydrotreating & 160 & 32404 & 0.8 & 1.02 & 4 \\
\hline
\end{tabular}


Table S24. Basic properties of coal. ${ }^{4}$

\begin{tabular}{ccc} 
Components & Properties \\
C & $66 \% \sim 68 \%$ \\
H & $4 \%$ \\
S & $<0.6 \%$ \\
N & $0.76 \%$ \\
O & $12 \%$ \\
Moisture & $<16 \%$ \\
Ash & $\leq 12 \sim 18 \%$ \\
Volatile & $23 \% \sim 25 \%$ \\
LHV & $\geq 5200 \sim 5500 \mathrm{cal} / \mathrm{g}$ \\
\hline
\end{tabular}




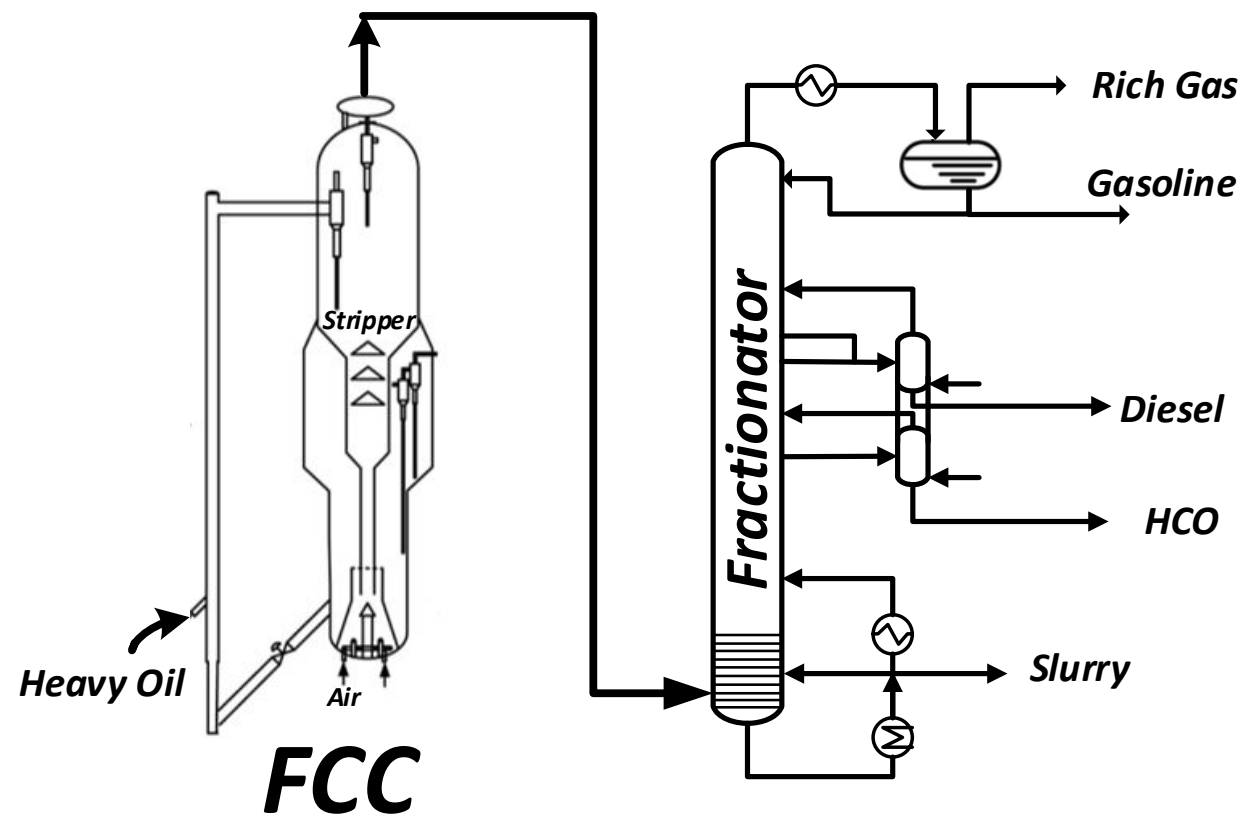

Figure S1. The process flowsheets of the FCC process. 


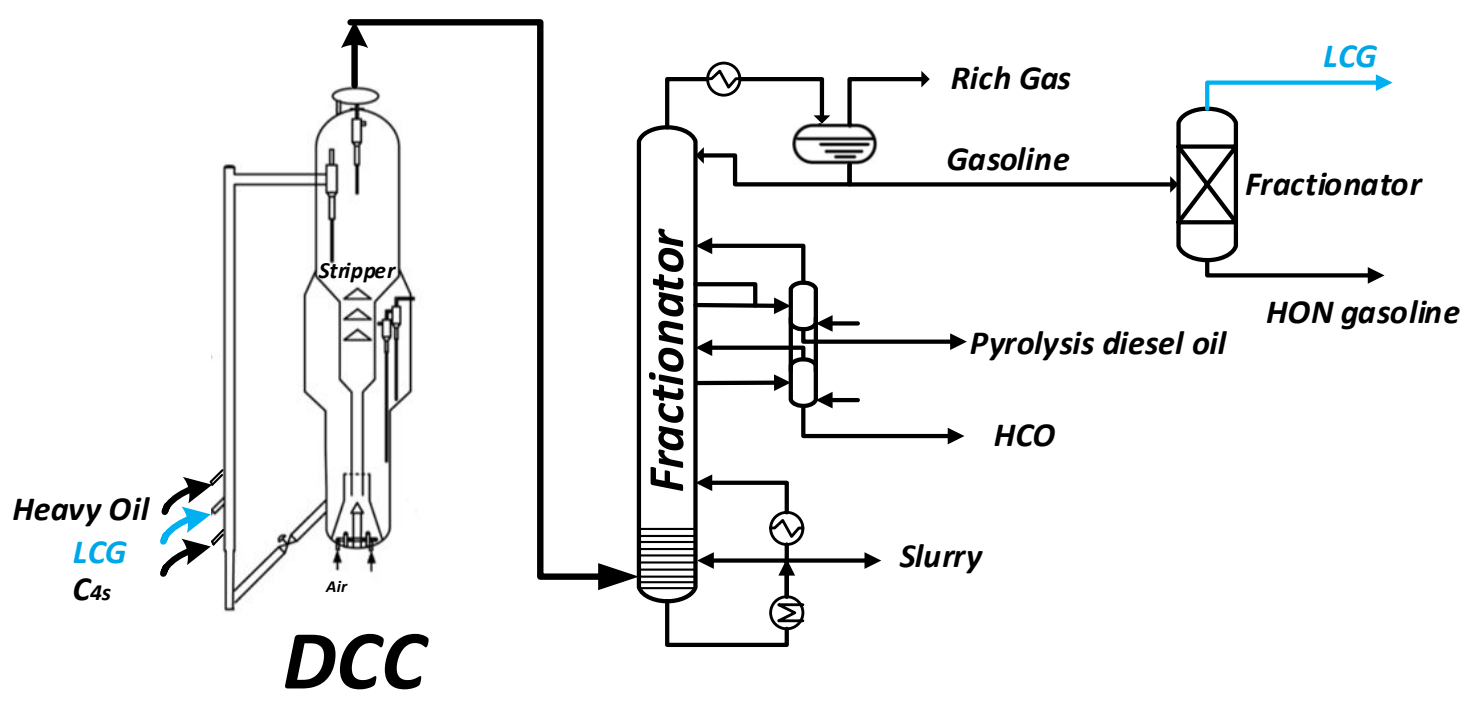

Figure S2. The process flowsheets of the DCC process. 


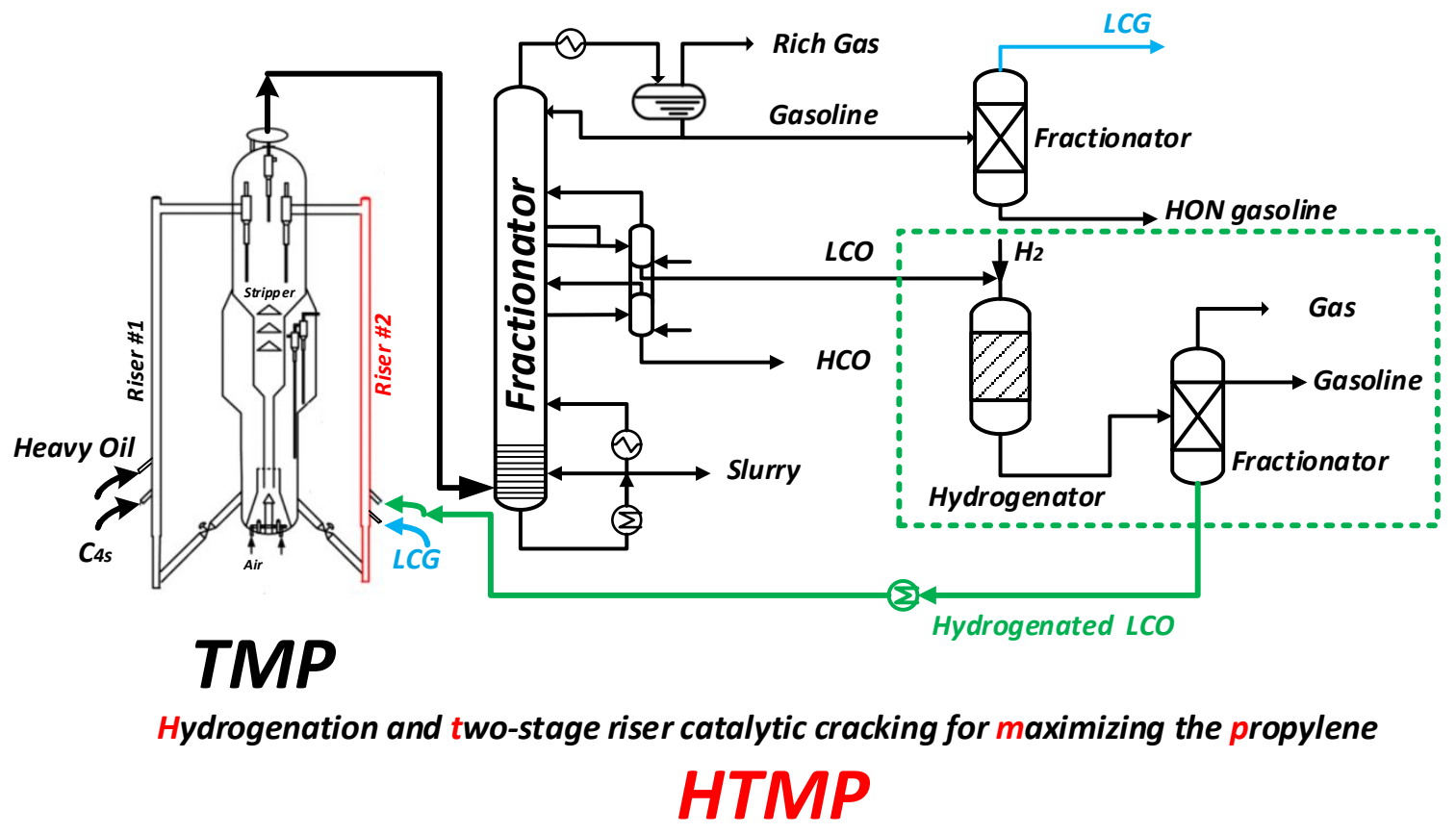

Figure S3. The process flowsheets of the HTMP process. 


\section{REFERENCES}

(1) Zou, R.; Luo, Q.; Mo, S. Study on kinetics of LGO pyrolysis and coke deposition. Petrol Proc Sec. 1991, 1, 1-8.

(2) Sinopec Economics \& Development Research Institute. Sinopec Project Feasibility Study Technical Economy-

Parameter \& Data. China Petrochemical Press: Beijing, 2018.

(3) Wang, X.; Shu, X. Heavy Oil Cracking to Light Olefins. China Petrochemical Press: Beijing, 2015.

(4) Li, D.; Nie, H.; Sun, L. Hydrotreating Process and Engineering. China Petrochemical Press: Beijing, 2016.

(5) Xu, C. Catalytic Reforming Process and Engineering. Process and Engineering. China Petrochemical Press: Beijing, 2014.

(6) He, K.; He, Y.; Wu, D. Technology and economy analysis of gasoline hydrogenation and aromatics extraction improvement process for ethylene complex. Petrochemical Technology Application. 2008, 26, 55-60. 